\title{
On the wavelength drift of spectral features from structured hot star winds
}

\author{
W.-R. Hamann ${ }^{1}$, J. C. Brown ${ }^{2}$, A. Feldmeier ${ }^{1}$, and L. M. Oskinova ${ }^{2}$ \\ 1 Professur Astrophysik, Universität Potsdam, Am Neuen Palais 10, 14469 Potsdam, Germany \\ 2 Department of Physics and Astronomy, University of Glasgow, Glasgow G12 8QQ, Scotland, UK
}

Received 27 July 2001 / Accepted 4 September 2001

\begin{abstract}
Spectral lines formed in stellar winds from OB stars are observed to exhibit profile variations. Discrete Absorption Components (DACs) show a remarkably slow wavelength drift with time. In a straightforward interpretation, this is in sharp contradiction to the steep velocity law predicted by the radiation-driven wind theory, and by semi-empirical profile fitting. In the present paper we re-discuss the interpretation of the drift rate. We show that the Co-rotating Interaction Region (CIR) model for the formation of DACs does not explain their slow drift rate as a consequence of rotation. On the contrary, the apparent acceleration of a spectral CIR feature is even higher than for the corresponding kinematical model without rotation. However, the observations can be understood by distinguishing between the velocity field of the matter flow, and the velocity law for the motion of the patterns in which the DAC features are formed. If the latter propagate upstream against the matter flow, the resulting wavelength drift mimics a much slower acceleration although the matter is moving fast. Additional to the DACs, a second type of recurrent structures is present in observed OB star spectra, the so-called modulations. In contrast to the DACs, these structures show a steep acceleration compatible with the theoretically predicted velocity law. We see only two possible consistent scenarios. Either, the wind is accelerated fast, and the modulations are formed in advected structures, while the DACs come from structures which are propagating upstream. Or, alternatively, steep and shallow velocity laws may co-exist at the same time in different spatial regions or directions of the wind.
\end{abstract}

Key words. stars: early-type - stars: winds, outflows - stars: mass-loss - stars: atmospheres - line: profiles

\section{Introduction}

When the UV spectral range became accessible in the 1970s, hot stars were found to have winds generally. Many studies of OB star since that time aimed at the determination of the basic properties of these winds, namely the mass-loss rate and the velocity law. Semi-empirical models were compared to the observed P Cygni profiles of the observed UV resonance lines and the $\mathrm{H} \alpha$ profile. Almost all studies employed the analytical $\beta$ law (see Eq. (2)) for a parametrized description of the velocity field. Generally it was found that with $\beta \approx 1$ velocity laws the observed spectra can be reproduced best (e.g. Puls et al. 1996), although the sensitivity of these methods to the form of the velocity law is limited.

The theory of radiation-driven winds in its original form (Castor et al. 1975) predicts as steady solution a $\beta$ velocity law with $\beta=1 / 2$. With the necessary correction for the "finite-disk effect", the numerical integration still gives a velocity field very close to a $\beta$ law with slightly

Send offprint requests to: W.-R. Hamann,

e-mail: wrh@astro.physik.uni-potsdam.de higher exponent, $\beta=0.8$ (Pauldrach et al. 1986; Friend $\&$ Abbott 1986). Ionization structure effects can in principle modify this result, but detailed numerical models for O star winds always arrived at velocity fields of similar form with $\beta$ being close to 0.8 (e.g. Puls et al. 1996).

With increasing observational material it was discovered that spectral lines formed in stellar winds exhibit profile variations. Thus it became clear that stellar winds are not smooth and stationary, but structured to some extent. Time series of spectra revealed that these short-time variations are not random, but show characteristic patterns which were termed "Discrete Absorption Components". These are superimposed on the unsaturated absorption part of P Cygni profiles and drift to higher blue-shift with time (e.g. Prinja \& Howarth 1988; Massa et al. 1995; Prinja et al. 1998; de Jong et al. 2001).

These drifting features open a diagnostic access to the wind kinematics. Already the first empirical studies of DACs concluded that their wavelength drift is remarkably slow. In a straightforward interpretation (which will be under debate in the rest of this paper), this is in sharp contradiction to a $\beta$ velocity law with $\beta \approx 1$. This poses 
the severe question of whether the radiation-driven wind theory as well as the semi-empirical spectroscopic studies are basically wrong. We mention in passing that emission lines in Wolf-Rayet spectra show also profile variations, which are found to contradict a steep $\beta=1$ velocity law (e.g. Lépine \& Moffat 1999).

After DACs turned out to repeat more or less periodically in many cases, this phenomenon was seen in relation to stellar rotation. Mullan (1984) proposed, in analogy to the solar wind, so-called Co-rotating Interaction Regions (CIRs) as the origin of the profile variability. This model has been worked out in some detail in kinematic studies (Owocki et al. 1995; Fullerton et al. 1997), and a first hydrodynamical simulation of CIRs in $2 \mathrm{D}$ was presented by Cranmer \& Owocki (1996). However, the CIR model alone does not help to resolve the contradiction between a fast velocity law and the apparently slow drift rate of DACs. Section 2 of the present paper is devoted to these questions.

Time-series observations of unprecedented quality, with respect to long duration and uninterrupted time coverage, have been obtained by the MEGA campaign cooperation (Massa et al. 1995) with the IUE satellite in 1995 for three hot stars, the prototype $\mathrm{O}$ supergiant $\zeta$ Puppis, one B0.5 star, and one Wolf-Rayet star. The OB stars actually show two different types of periodic line profile variations, the DACs already known before, and the socalled modulations. In Sect. 3 of this paper we will employ the MEGA observations of $\zeta$ Puppis in order to illustrate our kinematical considerations. A second, related paper (Brown et al., in prep.) will be focussed on the question, which information can be retrieved from observed DACs by the application of inverse methods.

\section{Simple kinematic modeling}

\subsection{Definitions}

In a spherically-symmetric stellar wind, the radial coordinate $r$ is expressed in units of the stellar radius $R_{*}$. Thus, $r>1$ in the wind. The velocity field of the wind, $v(r)$, is directed radially outward and written as the product of the terminal velocity $v_{\infty}$ and the dimensionless, normalized function $w(r) \leq 1$,

$v(r)=v_{\infty} w(r)$.

For our specific examples we will employ the well-known analytical velocity law which is commonly termed as the " $\beta$ law",

$w(r)=\left(1-\frac{1}{r}\right)^{\beta}$.

Time will be expressed by the dimensionless variable $t$, which is in units of flight time,

$t_{\text {flight }}=R_{*} / v_{\infty}$
Note that with these dimensionless quantities, the velocity $w$ is just

$w(r)=\frac{\mathrm{d} r}{\mathrm{~d} t}$

and hence the time $t$ (in units of flight times) is the integral

$t=\int \frac{\mathrm{d} r}{w}$

without any further factors. As we will show various plots of the function $t(w)$ in the following, we note here that the slope of such curves is the inverse of the acceleration,

$\frac{\mathrm{d} t}{\mathrm{~d} w}=\frac{1}{\ddot{r}}$.

\subsection{Advected patterns}

Let us assume there is some local structure, e.g. a density enhancement or rarefaction, or a small disturbance in the gradient of the velocity field, causing some excess or lack of line optical depth. Let us furthermore consider a UV resonance line, for which the absorption part of the emerging P Cygni profile is formed by line scattering between the stellar disk and the observer. For simplicity we consider only the central ray, i.e. we neglect the finite size of the stellar disk.

The postulated local narrow disturbance in optical depth can obviously produce a drifting narrow spectral feature superimposed on the stationary line profile. Its observed Doppler blue-shift reflects, of course, the velocity of the matter at the location of the pattern,

$\Delta \lambda=-w_{\text {mat }}(t) \Delta \lambda_{\mathrm{D}}$ with $\Delta \lambda_{\mathrm{D}}=\lambda_{0} v_{\infty} / c$.

Let us now assume, as a first step, that the local structure (pattern) is fully advected with the flow of matter. Consequently, the Doppler blue-shift of the corresponding spectral feature simply develops with time as described by Eq. (5) with $w=w_{\text {mat }}=w_{\text {pat }}$. Figure 1 displays, for later comparison, $t(w)$ for $\beta$ laws with $\beta=0.5,1,2$ and 4 . The analytic integrals are compiled in the Appendix.

\subsection{Propagating patterns}

However, it is by no means sure that any pattern superimposed on the mean velocity or density field is fully advected with the flow of matter. Abbott (1980) discovered a new type of hydrodynamic waves in line-radiation driven winds, later often termed Abbott waves. By means of these waves, a structure may propagate upstream against the flow of matter with supersonic speed, while the matter is passing through that pattern. The same holds for reverse shocks which form in a radiation driven wind due to an instability mechanism (Owocki et al. 1988). As noticed e.g. by Lamers (1994), the observed wavelength drift of features arising from such propagating patterns is not a direct measure of the acceleration of the wind matter.

Hence, in the reference frame of the star, the radial velocity $w_{\text {pat }}$ of a pattern might be considerably slower than 


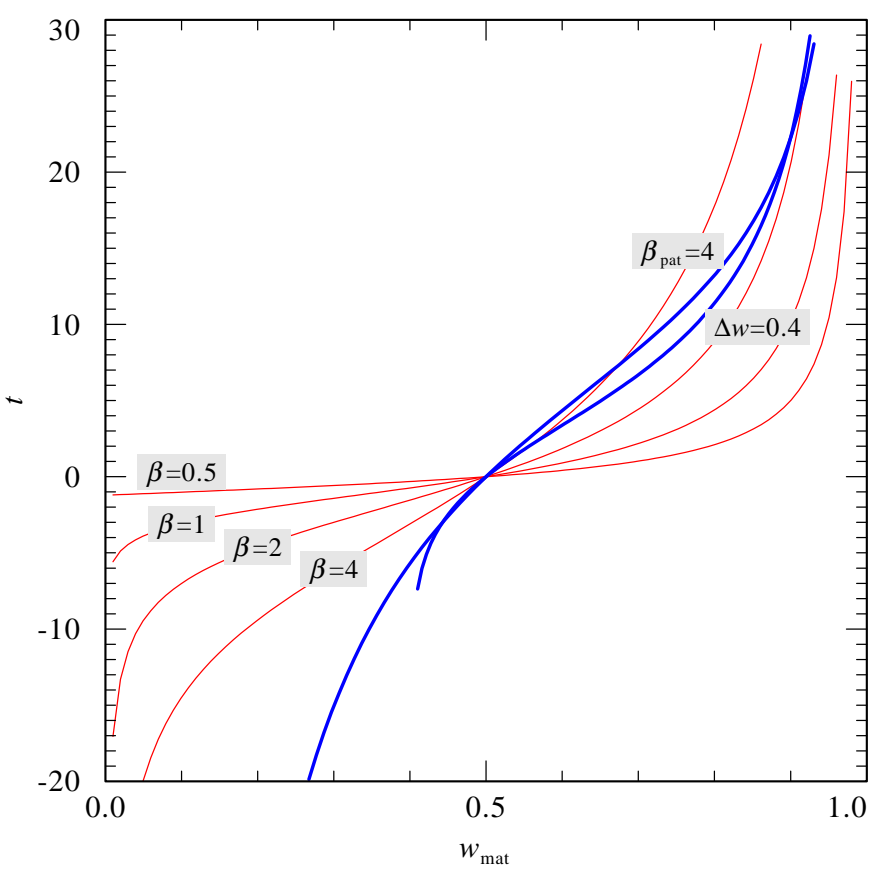

Fig. 1. Time $t$ until the matter producing a narrow spectral feature has reached the velocity $w_{\text {mat }}$. All curves are normalized at $t=0$ for $w_{\text {mat }}=0.5$. The thin curves are for the case that the pattern which gives rise to the spectral feature is fully advected with the flow of matter (i.e. $w_{\text {mat }}=w_{\text {pat }}$ ), and both are moving according to a $\beta$ velocity law (labels: $\beta$ ). The two thick curves take into account that the pattern where the narrow feature is formed may propagate upstream with respect to the matter flow. For the matter velocity the standard $\beta=1$ law is assumed in both examples, while the pattern moves with a different, slower velocity: either a $\beta=4$ law (label: $\beta_{\text {pat }}=4$ ), or a constant velocity difference relative to the matter flow (label: $\Delta w=0.4)$.

the matter velocity $w_{\text {mat }}$ at the same location. Cranmer \& Owocki (1996) in their two-dimensional hydrodynamic model obtained a structure (kink plus velocity plateau) in the radial velocity field which propagates outward roughly according to a $\beta$ law with exponent $\beta$ between 2 and 4 . Alternatively, one might suggest that the travel speed of the pattern relative to the matter is constant, i.e. $w_{\text {pat }}=w_{\text {mat }}-\Delta w$ (in order to obtain only outward traveling patterns, we restrict this definition to sufficiently large radii).

The effect of distinguishing between matter and pattern velocity is illustrated in Fig. 1 by two examples. In both cases, we adopt a $\beta_{\text {mat }}=1$ velocity law for $w_{\text {mat }}$. One of the thick lines gives $t\left(w_{\text {mat }}\right)$ assuming that $w_{\text {pat }}$ is following also a $\beta$ law, but with a different exponent $\beta_{\text {pat }}=4$. Thus the matter speed $w_{\text {mat }}$ and the pattern speed $w_{\text {pat }}$ at the same radius $r$ are related by $w_{\text {pat }}^{1 / \beta_{\text {pat }}}=w_{\text {mat }}^{1 / \beta_{\text {mat }}}$, which leads to the simple substitution

$w_{\mathrm{pat}}=w_{\mathrm{mat}}^{\beta_{\mathrm{pat}} / \beta_{\mathrm{mat}}}$

in Eq. (A.24). For the other thick line we assume that the pattern velocity is lower than the matter velocity at the same radius by a constant offset $\Delta w=0.4$ (see Appendix A.3 for the analytical formulae).

Figure 1 demonstrates that upstream propagating patterns can easily produce a very slow drift of the spectral feature, although the flow of matter itself follows the "fast" standard $\beta=1$ velocity law. Compared to the simple "advected patterns" model, the spectral drift rather resembles the $\beta=2$ or $\beta=4$ case.

\subsection{Co-rotating patterns}

\subsubsection{Definitions}

Observed "discrete absorption components" (DACs) are often found to repeat periodically. It is plausible to identify the repetition time with the star's rotation period (or an integer fraction of the latter). It has been suggested that there are structures at the stellar surface (magnetic spots, pulsation patterns) which cause an azimuthal dependence of wind density and velocity. Clearly, if the star rotates, any azimuthal variation of the radial wind velocity leads to interaction between streams of different speed and thus to the formation of "Co-rotating Interaction Regions" (CIRs) similar to what is known from the solar wind (cf. Mullan 1984; Cranmer \& Owocki 1996).

While this model obviously explains the periodicity of DACs, there was some subliminal suggestion that their slow wavelength drift is also a consequence of the stellar rotation. In the following we will make clear that this idea is wrong.

We denote the equatorial velocity of a rotating star by $v_{\text {rot }}$. Only the equatorial plane is considered for simplicity. Furthermore we define the velocity ratio

$\Omega_{0}=v_{\text {rot }} / v_{\infty}$.

Plausible values range from $\Omega_{0}=0.05$ (if $v_{\text {rot }}=$ $100 \mathrm{~km} \mathrm{~s}^{-1}$ and $v_{\infty}=2000 \mathrm{~km} \mathrm{~s}^{-1}$ ) to $\Omega_{0}=0.2$ for critical rotation $\left(v_{\text {rot }}=1 / \sqrt{2} v_{\text {esc }}\right.$ and assuming that $v_{\infty}$ is three times the escape velocity $v_{\text {esc }}-$ Abbott 1978).

\subsubsection{Pattern path}

Let us consider a pattern or parcel in the stellar wind at polar coordinates $\left(r, \phi_{\text {obs }}\right)$ in the observer's frame. The radial component of the velocity is specified by some velocity law $w_{\text {pat }}(r)$.

In the observer's frame, the azimuth angle of this parcel changes with the angular velocity $\Omega(r)$,

$\frac{\mathrm{d} \phi_{\mathrm{obs}}}{\mathrm{d} t}=\Omega(r)$.

Note that the derivative is with respect to the dimensionless time $t$ as defined in units of the flight time (Eq. (3)). In these dimensionless units, the angular velocity at the stellar radius is just $\Omega(r=1)=\Omega_{0}$. Assuming that angular momentum is conserved,

$\Omega(r)=\frac{\Omega_{0}}{r^{2}}$. 
Thus, integration over time $t$ yields

$\phi_{\mathrm{obs}}=\Omega_{0} \int \frac{\mathrm{d} t}{r^{2}}$

Time $t$ can be eliminated by substituting $\mathrm{d} t=w^{-1} \mathrm{~d} r$,

$\phi_{\text {obs }}(r)=\Omega_{0} \int \frac{\mathrm{d} r}{w r^{2}}$.

This equation describes the trajectories of a pattern or parcel in the observer's frame. Analytical formulae for $\beta$ laws with specific exponents are given in the Appendix. Results for typical parameters are shown in Fig. 2 and demonstrate that in this frame the motion is nearly radial even in the case of extremely fast rotation, as soon as the radial velocity has reached some fraction of the terminal speed.

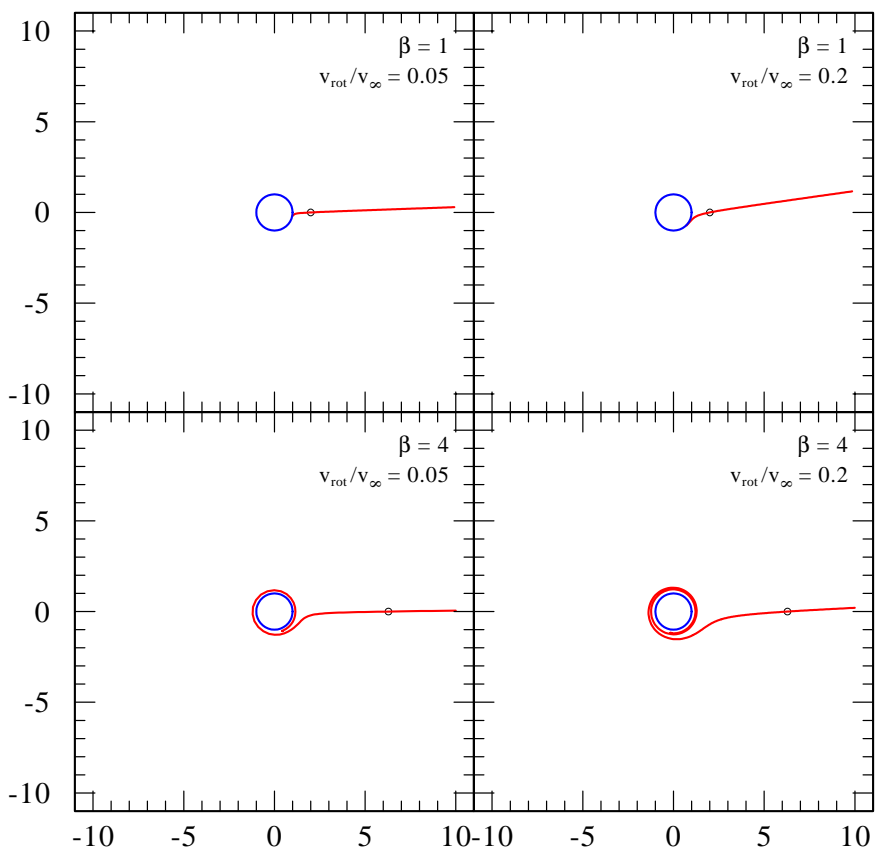

Fig. 2. Path of a parcel or pattern as seen in the observer's frame. The radial motion follows a $\beta$ law with exponents 1 and 4 (upper and lower frames, respectively). Conservation of angular momentum is assumed for the azimuthal motion, studying cases of slow and rapid rotation (left and right frames, respectively). The azimuth angle is normalized such that $\phi_{\text {obs }}=0$ when half the terminal velocity is reached $(w=0.5$, small circles).

Now we transform these trajectories from the observer's frame of reference into the co-rotating reference frame of the star. If we follow the motion of some pattern which at time $t_{\text {pat }}$ has the coordinates $\left(r, \phi_{\mathrm{obs}}\right)$, the azimuth angle in the co-rotating frame becomes

$\phi_{\text {cor }}(r)=-\Omega_{0} t_{\text {pat }}(r)+\phi_{\text {obs }}(r)$.

Figure 3 illustrates how the examples from Fig. 2 look after transformation into the co-rotating frame. Note that if $\Omega(r)$ decreases with $r$, as holds under any reasonable assumption, the angle $\phi_{\text {cor }}$ is decreasing with $r$ because the pattern lags back behind the stellar rotation.

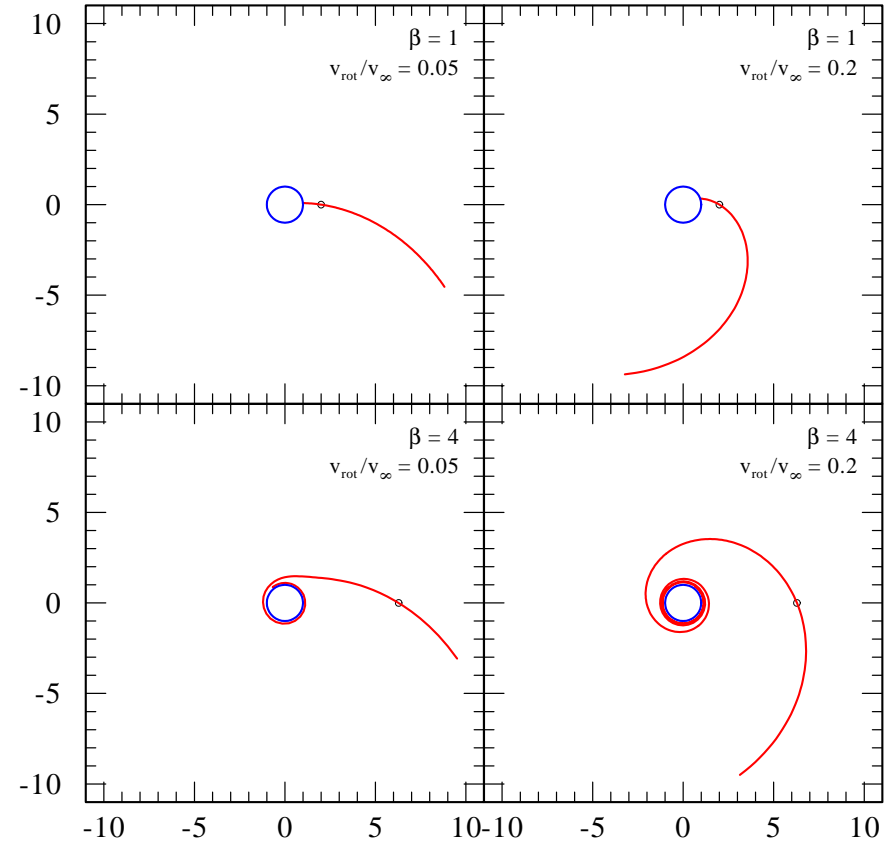

Fig. 3. Path of a pattern as seen in the co-rotating frame of the star. Otherwise, same as Fig. 2.

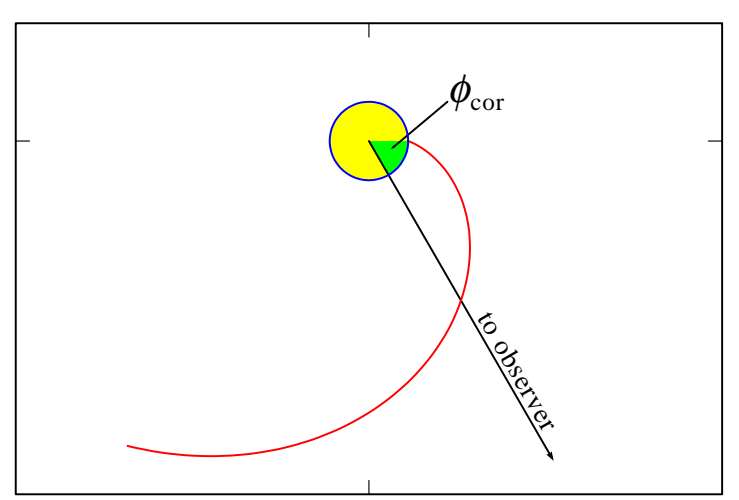

Fig. 4. Path of a pattern or parcel leaving the equator of a counter-clockwise rotating star, sketched in the frame corotating with the star. Due to the assumed conservation of angular momentum, the streamline lags back behind the rotation. In that frame, the azimuthal angle towards the observer changes as $-\Omega_{0} t$. A spectral feature thus may arise from the intersection point of the spiral structure and the ray towards the observer, and its Doppler shift reflects the matter velocity at this location.

\subsubsection{Wavelength drift}

As seen from the co-rotating frame of the star, the remote observer is circling around in a clockwise direction with angular frequency $\Omega_{0}$ (cf. Fig. 4). The line-of-sight (only considering the central ray) crosses the co-rotating spiral pattern. Let us denote by $t_{\text {pat, LS }}\left(w_{\text {pat }}\right)$ the time when the pattern velocity in this intersection point reaches $w_{\text {pat }}$. The azimuthal direction to the observer at this time is

$\phi_{\text {cor }}=-\Omega_{0} t_{\text {pat }, \mathrm{LS}}$ 
which may be inserted into Eq. (14) to obtain

$t_{\text {pat } \mathrm{LS}}\left(w_{\text {pat }}\right)=t_{\text {pat }}\left(w_{\text {pat }}\right)-\frac{1}{\Omega_{0}} \phi_{\text {obs }}\left(w_{\text {pat }}\right)$.

Note that we now consider all terms in this equation as being functions of $w_{\text {pat }}$. The time $t_{\text {pat }}$ until a pattern reaches velocity $w_{\text {pat }}$ is just the same function already discussed for the non-rotating case (Sects. 2.2 and 2.3) and is shown in Fig. 1. The term $\Omega_{0}^{-1} \phi_{\text {obs }}\left(w_{\text {pat }}\right)$ appeared already in the preceding section. The Eq. (13) for $\phi_{\text {obs }}$ contains a factor $\Omega_{0}$ which thus cancels out in Eq. (16). Hence the wavelength drift of a spectral feature, according to the CIR kinematics, does not depend on the rotation rate of the star.

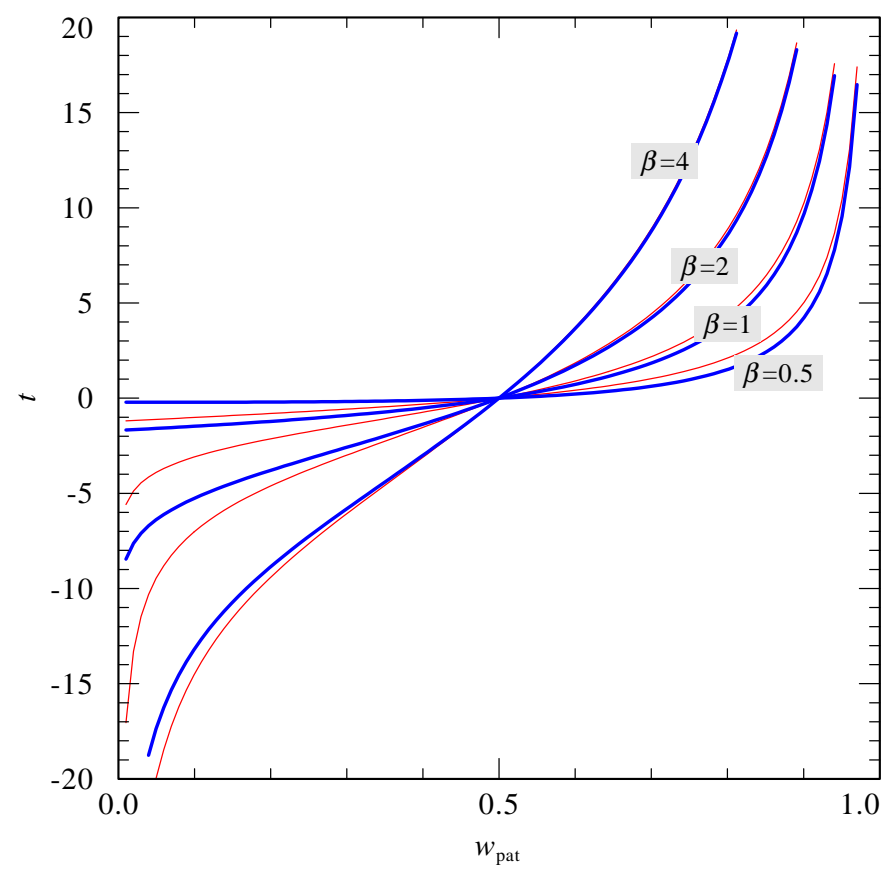

Fig. 5. Time $t$ until a pattern moving radially according to a $\beta$ law has reached the velocity $w_{\text {pat }}$. All curves are normalized at $t=0$ for $w_{\text {pat }}=0.5$. The thin curves are for the sphericallysymmetric model. The corresponding thick curves are for the CIR model, i.e. give the relation between $t_{\text {pat, LS }}$ and $w_{\text {pat }}$ at the intersection point between the co-rotating structure and the line-of-sight.

Moreover, the second term in Eq. (16) means subtracting a positive number because $\phi_{\mathrm{obs}}$ is positive (Eq. (13)) as long as $\Omega(r)$ remains positive. This holds not only for angular momentum conservation but for any reasonable assumptions. Figure 5 illustrates these relations for $\beta$ laws, the analytical formulae being given in the Appendix. The thin solid lines represent $t_{\text {pat }}$, i.e. the time until velocity $w_{\text {pat }}$ is reached in the spherically-symmetric model (same curves as in Fig. 1). The thick lines correspond to the CIR model and give $t_{\text {pat, LS }}$, i.e. the time until $w_{\text {pat }}$ is reached at the intersection point between the co-rotating pattern and the line-of-sight. The figure shows that in the CIR model the velocity is drifting even faster than in the nonrotating case with the same radial velocity law because of $t_{\text {pat LS }}<t_{\text {pat }}$. (For high $\beta$ the difference is only noticeable at low velocities.) In other words, the kinematics of the CIR model does not explain the observed slow drift of DACs on only kinematical grounds.

Although this result is implicitly contained in the literature (e.g. Fullerton et al. 1997), there was some confusion about this when e.g. Cranmer \& Owocki (1996) stated their surprise that in their models they find no correlation between the DAC's acceleration timescale and the star's rotation velocity. Similarly misleading, correlations between rotational velocity and the timescale of the DACs drift have been considered as straightforward evidence for the CIR model (Prinja 1988).

Summarizing, we have discussed two different effects which influence the wavelength drift of DACs: the propagation of patterns relative to the flow of matter, and the CIR geometry. The combination of both effects is straightforward (cf. Appendix). However, these two effects oppose each other. Only patterns which are upstreaming lead to a slower wavelength drift of the observable discrete features, while the CIR geometry makes them drift faster, independent of the rotation rate. While the latter effect is only mild, the former can be arbitrarily strong when the upstreaming velocity is high. In the hydrodynamical CIR simulation by Cranmer \& Owocki (1996) the "Abbott kink" propagating upstream causes the slow drift of the predicted DACs.

\section{Comparison with observations of $\zeta$ Pup}

Time-series observations of unprecedented quality, with respect to long duration and uninterrupted time coverage, have been obtained by the MEGA campaign cooperation (Massa et al. 1995) with the IUE satellite in 1995 for three hot stars, the prototype O-type supergiant $\zeta$ Puppis, the B0.5 supergiant HD 64760, and the Wolf-Rayet star WR 6. In the following we use the $\zeta$ Puppis data of the Si IV resonance doublet in order to illustrate the kinematical effects discussed in the preceding section. A thorough analysis of these observational data was given by Howarth et al. (1995), and we do not intend to add to their elaborate work.

In Figs. 6-8 we copy the grey-scale (or color) representation of the dynamical spectrum from Massa et al. (1995). It shows the difference between the individual observations and a "minimum absorption" template for the profile of the Si IV doublet at 1393.8, 1407.8 . The two vertical black lines mark the center wavelengths of the unshifted doublet components. We divide the abscissa axis in Doppler units referring to an adopted terminal wind velocity $v_{\infty}$ of $2300 \mathrm{~km} \mathrm{~s}^{-1}$.

Figure 6 is to clarify our terminology. Two types of dynamical features can be recognized in the data by eye, and have been quantified by the analyses by Howarth et al. (1995): the "DACs" repeat with a period of 5.21 days, which is presumably the rotation period of that star. These features only arise at high velocities, extending roughly over blueshift from -0.4 to -1.0 (Doppler units 


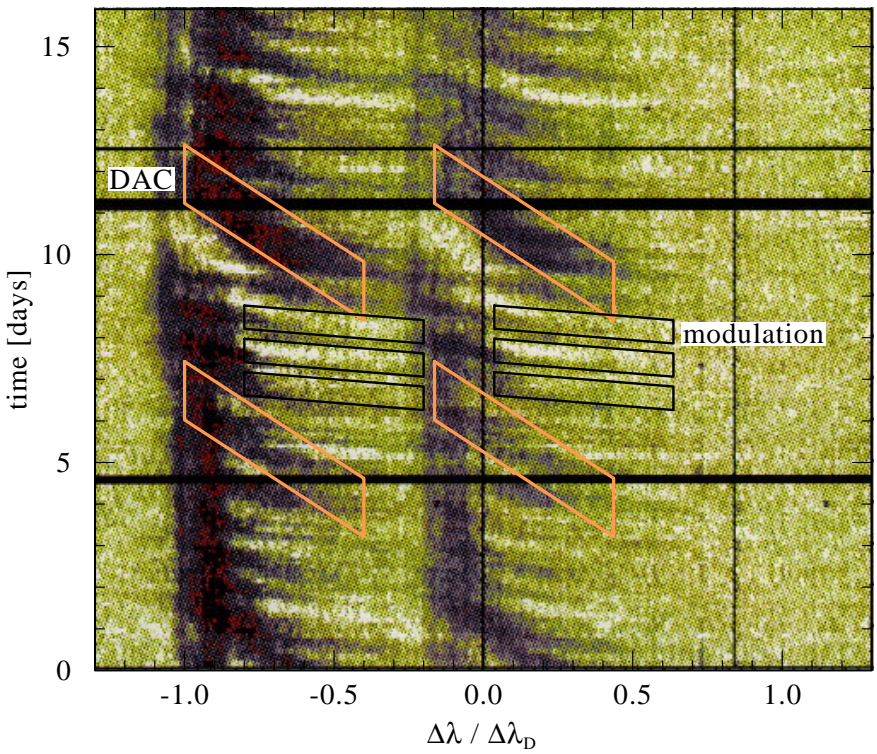

Fig. 6. Time variations in the SiIV resonance doublet of $\zeta$ Puppis, observed during the IUE MEGA campaign (Massa et al. 1995). The grey-scale (or color) representation is taken from that paper and shows the difference between the individual observations and a "minimum absorption template". The two vertical black lines mark the center wavelengths of the unshifted doublet components. The abscissa is divided in Doppler units referring to $v_{\infty}$ of $2300 \mathrm{~km} \mathrm{~s}^{-1}$, and with respect to the center wavelength of the blue doublet component. The boxes should clarify which kind of features we term "DACs" and "modulations". The boxes are drawn twice, according to the wavelength separation of the doublet components. The DACs boxes are repeated with the recurrence time of DACs $(5.21 \mathrm{~d}$, presumably the star's rotation period), while the modulations are indicated with their period of $19.2 \mathrm{~h}$.

of $\left.v_{\infty}\right)$. They show slow acceleration, which is reflected by the steep inclination of the corresponding boxes (cf. Eq. (6)). In the B supergiant HD 64760, another target of the MEGA campaign (Massa et al. 1995), the DAC structures are much more pronounced than in $\zeta$ Puppis.

The second type of structures is called "modulation" (Owocki et al. 1995). They repeat with a period of 19.2 hours, which is probably not an integer fraction of the rotation period (Howarth et al. 1995). One may speculate that they are triggered by (nonradial) pulsations, perhaps beats between neighboring mode frequencies. The modulations are most pronounced at intermediate blueshift ( -0.2 to -0.8 Doppler units), and drift fast. The modulations are not "discrete" absorption components, but rather broad and shallow. Therefore we suggest not to term them "DACs".

Now the observed dynamical spectrum from Fig. 6 is overplotted with curves for the wavelength drift calculated from the simple kinematical models discussed in Sect. 2. In Fig. 7 we assume the $\beta=1$ velocity law and a pattern which is fully advected with the flow (Sect.2.2 and Eq. (A.10)). The curves are drawn twice for the appropriate center-wavelengths of each doublet component. In time they are plotted repeatedly with the "modulation" period

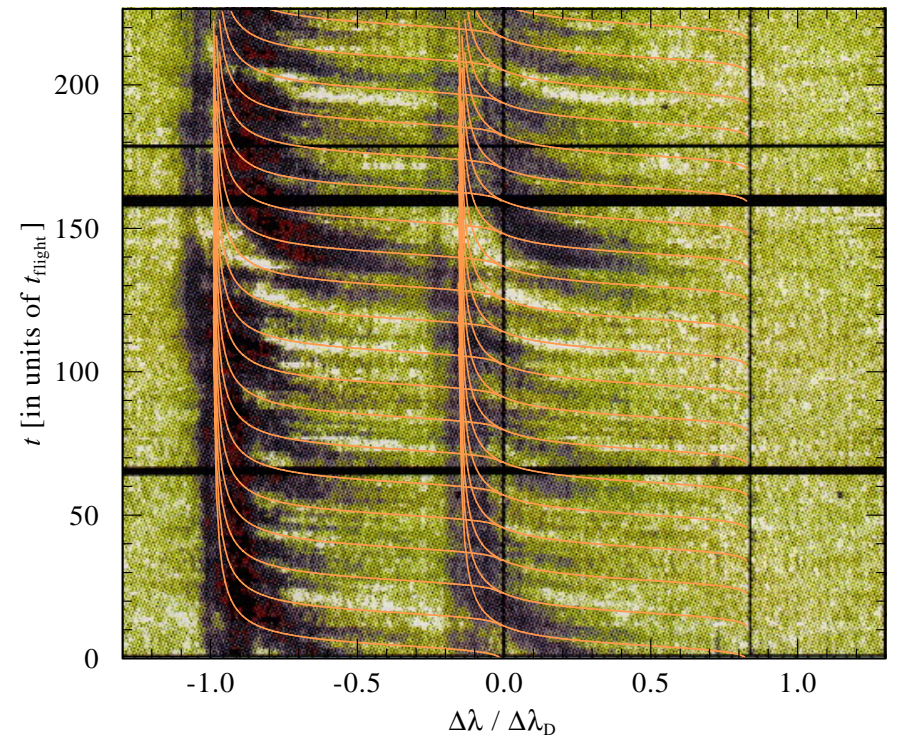

Fig. 7. Same observations as displayed in Fig. 6, now overplotted with the wavelength drift calculated for a $\beta=1$ velocity law and a pattern which is fully advected with the flow (Sect. 2.2; Eq. (A.10)). The curves are drawn twice with appropriate shift between the doublet components, and repeated with the period of the "modulations" of $19.2 \mathrm{~h}$. The time at the ordinate axis, spanning the whole observation run of 15.9 days, is expressed in units of the flight time $t_{\text {flight }}=R_{*} / v_{\infty}=1.68 \mathrm{~h}$. The slope of the modulation patterns is not in conflict with this "fast" velocity law.

of $19.2 \mathrm{~h}$. Note that the time at the ordinate axis, spanning over the whole observation run of 15.9 days, is now expressed in units of the flight time $t_{\text {flight }}=R_{*} / v_{\infty}=1.68 \mathrm{~h}$, adopting a stellar radius of $R_{*}=20 R_{\odot}$ and terminal velocity of $v_{\infty}=2300 \mathrm{~km} \mathrm{~s}^{-1}$.

The rough by-eye comparison reveals that the slope of the modulations is not in conflict with the calculated curves. Hence there is no need to invoke any upstreamtraveling patterns, and the "fast" $\beta \approx 1$ velocity law promoted by the radiation-driven wind theory is fine.

Alternatively, attributing the modulations to CIRs would make the slope even a bit smaller, which mimics a slightly faster acceleration (cf. Fig. 5). Hence the $\beta=1$ velocity law and fully advected patterns remain compatible with the slope of the modulations in the CIR case as well, at least at velocities $w>0.4$ where the modulations are visible, though at smaller $w$ the predicted curves differ. In fact, detailed studies of the "banana shaped" modulations observed in the B type star HD 64760 (Fullerton et al. 1997) explained even the "phase bowing" within the framework of the CIR model, as a consequence of the finite stellar disk.

In contrast to the modulations, the DACs which are weakly visible in the $\zeta$ Puppis data show a much slower drift rate. In Fig. 8 the $\zeta$ Pup observations are overplotted with the wavelength drift calculated for a narrow feature arising from a pattern which propagates upstream with a relative velocity of $0.4 v_{\infty}$, while the matter flows with a $\beta=1$ velocity law (Sect. 2.3 and Eq. (A.16)). The plotted 


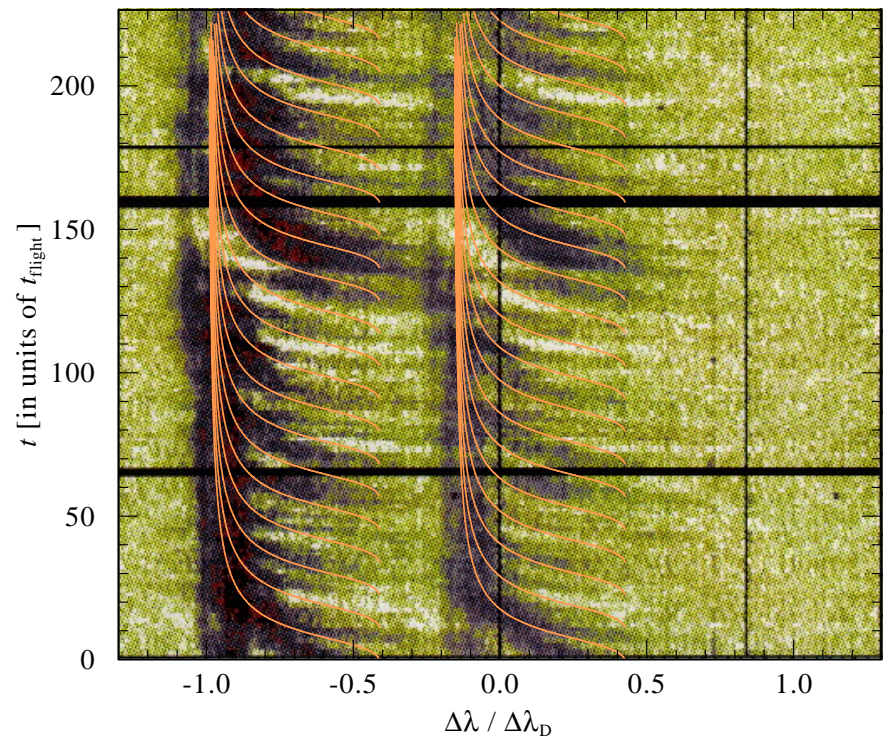

Fig. 8. Same as Fig. 7, now overplotted with the wavelength drift calculated for a narrow feature arising from a pattern which propagates upstream with a relative velocity of $0.4 v_{\infty}$, while the matter flows with a $\beta=1$ velocity law (Sect. 2.3; Eq. (A.16)). The slope is much steeper now (i.e., the acceleration is smaller) than the "modulation" pattern, but comparable with the slow motion of the DACs. The curves are repeated with the frequency of the "modulations" of $19.2 \mathrm{~h}$, while the DACs recurrence period is 5.21 days (cf. Fig. 6).

curves again repeat with the frequency of the "modulations" of $19.2 \mathrm{~h}$, while the DACs recurrence period is actually 5.21 days (cf. Fig. 6).

As Fig. 8 reveals, the calculated curves show much steeper slopes than the observed "modulation" pattern, but roughly reproduce the steeper slope of the DACs. This demonstrates the capability of the "upstreaming pattern" scenario to explain their slow apparent acceleration.

\section{Conclusions}

In time-resolved spectroscopy of UV resonance lines from hot star winds, different types of recurrent features (DACs and modulations) are superimposed on the unsaturated absorption part of P Cygni profiles. The wavelength drift rates of the DACs were stated to be in conflict with theoretically predicted wind velocity laws. In the present paper we re-discussed these drift rates on the basis of simple kinematical models.

In the simple picture that the drifting spectral features originate from wind structures (patterns) which are fully advected with the flow of matter, the slow wavelength drift observed for DACs can only be explained by adopting a shallow velocity law. In terms of the usual analytical $\beta$ velocity law, this implies a large exponent $\beta>2$. However, high $\beta$ values are in conflict with the radiation-driven wind theory which predicts $\beta \approx 1$, and are also not supported by detailed profile fits from line formation calculations.

The other possibility to explain small drift rates is by distinguishing between the velocity field of the matter flow, and the velocity law for the motion of the patterns in which the narrow spectral features form. If the latter propagate upstream against the matter, the resulting wavelength drift can mimic a much slower acceleration although the matter is still moving according to a low $\beta$.

In a rotating star, wind structures will lead to the formation of Co-rotating Interaction Regions (CIRs). There was some misunderstanding that absorption features arising from these regions will mimic a slower acceleration, depending on the rotation rate. Instead, we showed that in the CIR model the apparent acceleration is always steeper than derived from the non-rotating wind model with the same velocity law in the radial direction. This apparent additional acceleration does not depend at all on the rotation rate.

In addition to the DACs, a second type of periodic features are found in the dynamical OB star spectra, the so-called modulations. They show a steep acceleration in accord with $\beta \approx 1$, no matter whether the CIR model or a non-rotating model is considered. The presence of these modulations excludes models in which the whole wind is accelerated slowly (high $\beta$ ), and supports the theoretical prediction of $\beta \approx 1$. We see only two possibilities to reconcile DACs and modulations in one consistent picture. Either the wind is flowing fast, and the modulations are formed in advected structures, while the DACs come from structures which are propagating upstream. Or, alternatively, slow and fast velocity laws co-exist at the same time in different spatial parts of the wind, where fast streams follow the "critical" solution of the radiation-driven wind theory with $\beta \approx 1$ and exhibit modulations, while other parts (very dense clouds?) may adopt "overloaded" solutions and produce DACs.

Acknowledgements. We thank A. Fullerton for his interesting remarks, and for sending us the MEGA data. J. C. B. and L. M. O. acknowledge the support of PPARC research grants.

\section{Appendix A: Analytical integrals for $\beta$ laws}

Here we give analytical solutions for the wavelength drifts discussed in the main text, if the radial motion follows a $\beta$ velocity law. The following integrals are given, always dropping the integration constants:

- the time $t_{\text {pat }}$ a pattern needs to reach $w_{\text {pat }}$, i.e.

$$
t_{\text {pat }}=\int \frac{\mathrm{d} r}{w_{\text {pat }}}
$$

- the azimuthal angle $\phi_{\text {obs }}\left(w_{\text {pat }}\right)$ in the observer's frame, for a pattern which started from a footpoint at the stellar surface and traveled radially with $w_{\text {pat }}$, and azimuthally under conservation of angular momentum,

$$
\phi_{\mathrm{obs}}=\Omega_{0} \int \frac{\mathrm{d} r}{w_{\text {pat }} r^{2}} ;
$$

- the time $t_{\text {pat, LS }}$ until the radial speed of a pattern, measured in the place where the co-rotating structure 
cuts with the line-of-sight (LS) to the observer, reaches the value $w_{\text {pat }}$

$$
t_{\mathrm{pat}, \mathrm{LS}}=t_{\mathrm{pat}}-\frac{1}{\Omega_{0}} \phi_{\mathrm{obs}}
$$

Note that the observed Doppler shift of a spectral feature reflects the matter velocity $w_{\text {mat }}$ in the place of the pattern; these might differ, if the pattern represents e.g. a traveling wave or (reverse) shock. If both $w_{\text {mat }}$ and $w_{\text {pat }}$ can be approximated by $\beta$ laws with different $\beta_{\text {mat }}, \beta_{\text {pat }}$ respectively, $w_{\text {pat }}$ has to substituted in the final formulae by

$w_{\mathrm{pat}}=w_{\mathrm{mat}}^{\beta_{\mathrm{pat}} / \beta_{\mathrm{mat}}}$.

\section{A.1. Special case $\beta=0.5$}

$$
\begin{aligned}
& w_{\text {pat }}(r)=\left(1-\frac{1}{r}\right)^{\frac{1}{2}} \\
& -t_{\text {pat }}=\frac{w_{\text {pat }}}{1-w_{\text {pat }}^{2}}+\frac{1}{2} \ln \frac{1+w_{\text {pat }}}{1-w_{\text {pat }}} \\
& -\phi_{\text {obs }}=2 \Omega_{0} w_{\text {pat }} \\
& -t_{\text {pat }, \mathrm{LS}}=\frac{w_{\text {pat }}}{1-w_{\text {pat }}^{2}}+\frac{1}{2} \ln \frac{1+w_{\text {pat }}}{1-w_{\text {pat }}}-2 w_{\text {pat }} .
\end{aligned}
$$

\section{A.2. Special case $\beta=1$}

$$
\begin{aligned}
& w_{\text {pat }}(r)=1-\frac{1}{r} \\
& -t_{\text {pat }}=\frac{1}{1-w_{\text {pat }}}+\ln \frac{w_{\text {pat }}}{1-w_{\text {pat }}} \\
& -\phi_{\text {obs }}=\Omega_{0} \ln \left(w_{\text {pat }}\right) \\
& -t_{\text {pat }, \text { LS }}=\frac{1}{1-w_{\text {pat }}}+\ln \frac{1}{1-w_{\text {pat }}} .
\end{aligned}
$$

\section{A.3. Special case $\beta=1$, but with velocity offset}

$w_{\text {pat }}(r)=1-\frac{1}{r}-\Delta w$

considering only large enough radii where $w_{\text {pat }}(r)>0$.

$$
-t_{\mathrm{pat}}=(1-\Delta w)^{-2}[u+\ln (u)]
$$

where

$u=\frac{1-\Delta w}{1-w_{\text {pat }}-\Delta w}$.

One may define $\tilde{w}=w_{\text {pat }} /(1-\Delta w)$, which results in $u=1 /(1-\tilde{w})$ and then yields an expression for $t_{\text {pat }}$ which has a similar form as for the normal $\beta=1$ law without offset (Eq. (A.10)):

$$
t_{\text {pat }}=(1-\Delta w)^{-2}\left[\frac{1}{1-\tilde{w}}+\ln \frac{\tilde{w}}{1-\tilde{w}}\right]
$$

$$
\begin{aligned}
& -\phi_{\text {obs }}=\Omega_{0} \ln \left(w_{\text {pat }}\right) \\
& -t_{\text {pat }, \mathrm{LS}}= \\
& \quad(1-\Delta w)^{-2}\left[\frac{1}{1-\tilde{w}}+\ln \frac{\tilde{w}}{1-\tilde{w}}\right]-\ln \left(w_{\text {pat }}\right) .
\end{aligned}
$$

Assuming that the pattern is traveling "upstream" against the matter flow with relative velocity $\Delta w$, i.e. the matter follows the normal $\beta=1$ law without offset, implies $\tilde{w}=$ $\left(w_{\text {mat }}-\Delta w\right) /(1-\Delta w)$.

\section{A.4. Special case $\beta=2$}

$$
\begin{aligned}
& w(r)=\left(1-\frac{1}{r}\right)^{2} \\
& -t_{\text {pat }}=u+2 \ln u-\frac{1}{u} \\
& \quad \text { with } u=r-1=1 /\left(w^{-\frac{1}{2}}-1\right) \\
& -\phi_{\text {obs }}=-\Omega_{0} \frac{1}{u} \\
& -t_{\text {pat }, \mathrm{LS}}=u+2 \ln u .
\end{aligned}
$$

\section{A.5. Special case $\beta=4$}

$$
\begin{aligned}
& w_{\text {pat }}(r)=\left(1-\frac{1}{r}\right)^{4} \\
& -t_{\text {pat }}=u+4 \ln u-6 u^{-1}-2 u^{-2}-\frac{1}{3} u^{-3} \\
& \text { where } u=r-1=1 /\left(w_{\text {pat }}^{-\frac{1}{4}}-1\right) . \\
& -\phi_{\text {obs }}=-\Omega_{0}\left(u^{-1}+u^{-2}+\frac{1}{3} u^{-3}\right) \\
& -t_{\text {pat }, \text { LS }}=u+4 \ln u-5 u^{-1}-u^{-2} .
\end{aligned}
$$

\section{References}

Abbott, D. C. 1978, ApJ, 225, 893

Abbott, D. C. 1980, ApJ, 242, 1183

Cranmer, S. R., \& Owocki, S. P. 1996, ApJ, 462, 469

Friend, D. B., \& Abbott, D. C. 1986, ApJ, 311, 701

Fullerton, A. W., Massa, D. L., Prinja, R. K., Owocki, S. P., \& Cranmer, S. R. 1997, A\&A, 327, 699

Howarth, I. D., Prinja, R. K., \& Massa, D. 1995, ApJ, 452, L65 de Jong, J. A., Henrichs, H. F., Kaper, L., et al. 2001, A\&A, 368,601

Lamers, H. J. G. L. M. 1994, ASpSc, 221, 41

Lépine, S., \& Moffat, A. F. M. 1999, ApJ, 514, 909

Massa, D., Fullerton, A. W., Nichols, J. S., et al. 1995, ApJ, $452, \mathrm{~L} 53$

Mullan, D. J. 1984, ApJ, 283, 303

Owocki, S. P., Castor, J. I., \& Rybicki, G. B. 1988, ApJ, 335, 914

Owocki, S. P., Cranmer, S. R., \& Fullerton, A. W. 1995, ApJ, 453, L37

Prinja, R. K. 1988, MNRAS, 231, 21P

Prinja, R. K., \& Howart, I. D. 1988, MNRAS, 233, 123

Prinja, R. K., Massa, D., Howart, I. D., \& Fullerton, A. W. 1998, MNRAS, 301, 926

Pauldrach, A., Puls, J., \& Kudritzki, R. P. 1986, A\&A, 164, 86 Puls, J., Kudritzki, R.-P., Herrero, A., et al. 1996, A\&A, 305, 171 